\title{
Reflection coefficient for a lossy liquid-lossless isotropic solid interface
}

\author{
Abdullah Atalar \\ Edward L. Ginzton Laboratory, Stanford University, Stanford, California 94305 \\ (Received 4 October 1978) \\ A theoretical treatment of the reflection problem at a lossy liquid-lossless isotropic solid is given. The \\ calculation results indicate that the reflection coefficient has a peak greater than unity. A physical \\ explanation is proposed which also leads to the prediction that at some critical liquid attenuation a \\ Rayleigh wave propagating on the solid surface will not leak into the liquid.
}

PACS numbers: 43.20.Fn, 68.25. $+\mathrm{j}, 68.45 .+\mathrm{w}$

\section{INTRODUCTION}

The reflection coefficient for a plane wave obliquely incident at a plane interface between a liquid and a solid has been intensively studied. The solution for the lossless case can be found in some texts such as that by Brekhovskikh. ${ }^{1}$ The same problem for the lossless liquid-lossy solid has also been the subject of several papers. $^{204}$ Experimentally observed dip at the Rayleigh angle has been explained by the presence of loss in the solid. At high frequences liquid loss becomes significant $^{5}$ and therefore enters the problem. In this paper a treatment of the problem for lossy liquid-lossless solid will be given.

\section{THEORY}

Let us assume that a plane wave propagating in the $X Y$ plane is obliquely incident at a liquid-solid interface at $z=0$ as shown in Fig. 1. Let the incident wave be represented by its particle velocity field:

$$
\begin{aligned}
\bar{v}^{I}= & \left(\hat{x} \sin \theta_{I}+\hat{z} \cos \theta_{I}\right) \exp \left[j\left(k_{0}+j \alpha\right)\right. \\
& \left.\times\left(x \sin \theta_{I}+z \cos \theta_{I}\right)\right],
\end{aligned}
$$

where $\theta_{l}, k_{0}\left(=\omega / V_{0}\right)$ and $\alpha$ are the incidence angle, wavenumber and viscous damping in the liquid, respectively. Here $\exp (-j \omega t)$ time dependence is ignored. Aiso, let the reflected and refracted waves be shown by their velocity fields

$$
\begin{aligned}
\bar{J}^{R}= & R\left(\hat{x} \sin \theta_{I}-\hat{z} \cos \theta_{I}\right) \exp \left[j\left(k_{0}+j \alpha\right)\right. \\
& \left.\times\left(x \sin \theta_{I}-z \cos \theta_{I}\right)\right], \\
\bar{v}^{L}= & L\left(\hat{x} \sin \theta_{L}+\hat{z} \cos \theta_{L}\right) \\
& \exp \left[j k_{L}\left(x \sin \theta_{L}+z \cos \theta_{L}\right)\right], \\
\bar{J}^{S}= & S\left(-\hat{x} \cos \theta_{S}+\hat{z} \sin \theta_{S}\right) \\
& \exp \left[j k_{S}\left(x \sin \theta_{S}+z \cos \theta_{S}\right)\right],
\end{aligned}
$$

where $\theta_{L}, \theta_{S}, k_{L}\left(=\omega / V_{L}\right), k_{S}\left(=\omega / V_{S}\right)$ are the ref raction angle and wavenumber of the longitudinal and shear waves.

Using the boundary conditions for the velocity and stress at $z=0$ plane, one can find

$$
R=\frac{Z_{L} \cos ^{2} 2 \theta_{S}+Z_{S} \sin ^{2} 2 \theta_{S}-Z_{0}}{Z_{L} \cos ^{2} 2 \theta_{S}+Z_{S} \sin ^{2} 2 \theta_{S}+Z_{0}},
$$

where

$$
Z_{0}=\frac{\rho_{0} V_{0}}{\cos \theta_{I}\left[1+j\left(\alpha V_{0} / \omega\right)\right]},
$$

$$
\begin{aligned}
& Z_{L}=\frac{\rho V_{L}}{\cos \theta_{L}}, \\
& Z_{S}=\frac{\rho V_{S}}{\cos \theta_{S}}
\end{aligned}
$$

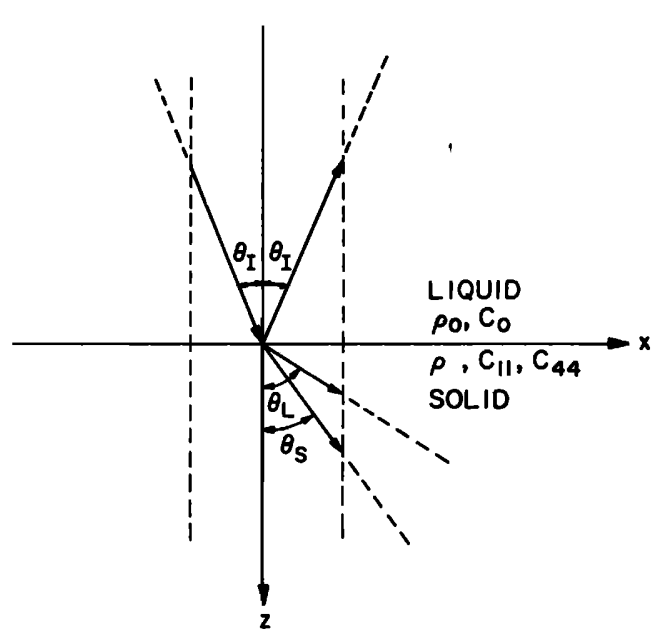

FIG. 1. Acoustic plane wave scattering at a plane boundary between a liquid and an isotropic solid. 
Note that these expressions can be obtained from the lossless results ${ }^{1}$ by changing $k_{0}$ with $k_{0}+j \alpha$.

Snell's Law takes the form

$$
\left[\left(1+j \frac{\alpha V_{0}}{\omega}\right)\left(V_{0}\right)^{-1}\right] \sin \theta_{I}=\frac{\left(\sin \theta_{L}\right)}{V_{L}}=\frac{\left(\sin \theta_{S}\right)}{V_{S}}
$$

To satisfy this equation $\sin \theta_{L}$ and $\sin \theta_{S}$ must be complex and therefore $\cos \theta_{L}$ and $\cos \theta_{S}$ have to be $\operatorname{complex}$ as well:

$$
\cos \theta_{i}=\mp\left[1-\left(\frac{V_{i}}{V_{0}}\right)^{2} \sin ^{2} \theta_{I}\left(1+j \frac{\alpha V_{0}}{\omega}\right)^{2}\right]^{1 / 2},
$$

for $i=L, S$.

Examining the forms of the refracted waves in Eqs. (3) and (4) helps us select the proper sign for $\cos \theta_{i}$. For $\sin \theta_{I}<\left(V_{0} / V_{i}\right), \cos \theta_{i}$ must have a positive real part so that the ref racted waves have a $k$ vector with a positive $z$ component. On the other hand, for $\sin \theta_{I}$ $>\left(V_{0} / V_{i}\right), \cos \theta_{i}$ has to have a positive imaginary part. This condition makes sure that the refracted waves die out as $z$ goes to infinity. Since $\cos \theta_{i}$ changes sign at the critical angles while its magnitude is nonzero, one may expect discontinuities in the reflection coefficient.

\section{RESULTS}

An HP9820A calculator program has been written to perform the numerical calculations summarized above. Figure 2 shows the reflection coefficient amplitude and phase for the water-YAG interface at 1, 2, 4, and 8 GHz. (We assume that $\alpha / f^{2}=22 \times 10^{-15}$ for water ) Notice that the reflection coefficient magnitude for incident angles near Rayleigh angle is greater than one! This peculiar behavior can be explained by the "Schoch displacement." The Schoch displacement is the acoustical counterpart of the optical "Goos-Hänchen shift." This phenomena is associated with the reflection of a wellcollimated beam incident on an interface at the Rayleigh critical angle. The reflected beam undergoes a sizeable lateral shift from the position predicted by geometrical considerations. This displacement is actually created by the reradiation of the Rayleigh wave from the region outside that ensonified by the incident beam. For the lossless case an analytical expression can be obtained for this lateral shift, $\Delta_{s}{ }^{6}$

$$
\begin{aligned}
\Delta_{s} & =-\left.\frac{1}{k_{0} \cos \theta_{I}} \frac{d\lfloor R}{d \theta_{I}}\right|_{\theta_{I} \theta_{R}} \\
& =\frac{2 \lambda_{0}}{\pi} \frac{\rho}{\rho_{0}} \frac{r(r-s)}{s(s-1)}^{1 / 2} \frac{1+6 s^{2}(1-q)-2 s(3-2 q)}{s-q},
\end{aligned}
$$
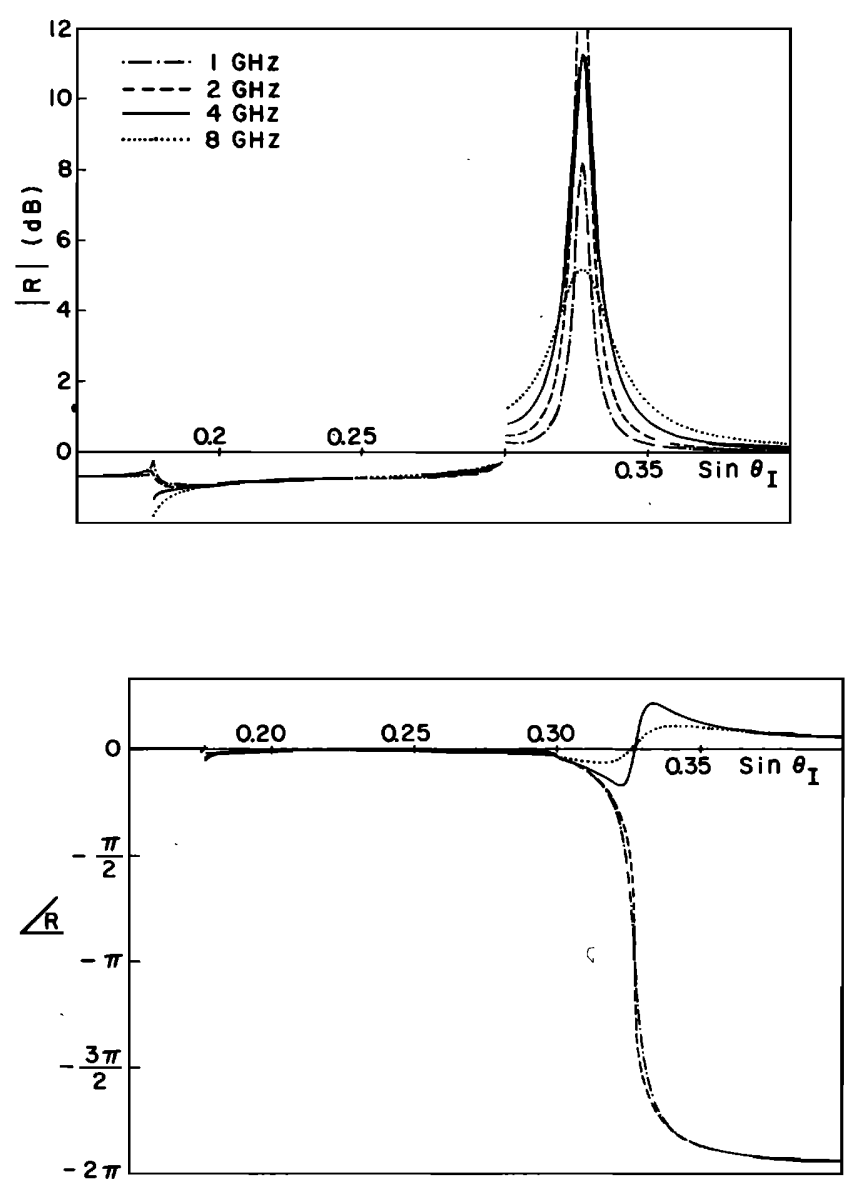

FIG. 2. Amplitude and phase of the reflection coefficient for $\mathrm{H}_{2} \mathrm{O}$-YAG interface at $1,2,4$, and $8 \mathrm{GHz}$.

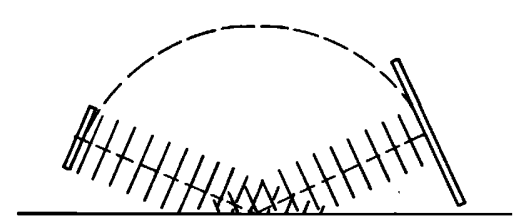

(a)

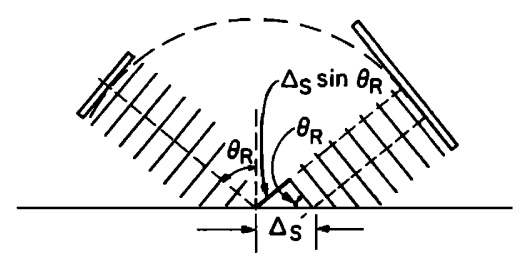

(b)

FIG. 3. Illustration of a setup to measure the reflection coefficient (a) at an angle greater than the Rayleigh angle, (b) at the Rayleigh angle. The liquid path in (b) is less than that in (a) by an amount $\Delta_{s} \sin \theta_{R}$. 
where

$$
\begin{aligned}
& s=\left(V_{s} / V_{R}\right)^{2} \\
& r=\left(V_{s} / V_{0}\right)^{2} \\
& q=\left(V_{s} / V_{L}\right)^{2} .
\end{aligned}
$$

Here $V_{R}$ represents the Rayleigh wave velocity.

We will assume that $\Delta_{s}$ is still given by Eq. (5) under small loss conditions. This is a good approximation, because the phase of the reflection coefficient changes very slightly with the introduction of small losses. ${ }^{2}$ Figure 3 depicts a setup to measure the reflection coefficient of the interface at different angles. If the liquid medium is lossy, the attenuation in the liquid path must be accounted for, and the measured reflection coefficient has to be normalized accordingly. In Fig。 3(a) an incidence angle larger than the Rayleigh angle with a liquid path of $2 d$ is shown. In this case the lateral shift is negligible and we obtain a unity reflection coefficient after normalization. In (b) the incidence angle is the Rayleigh angle. Therefore a lateral displacement will take place, and the liquid path will be reduced by $\Delta_{s}$ $\sin \theta_{R}$, but for normalization the full $2 d$ length is used. In this case we expect to obtain a reflection coefficient larger than unity by an amount determined by the attenuation in the path $\Delta_{s} \sin \theta_{R}$.

The reflection problem involving a lossless liquid and a lossy solid is discussed in the literature. ${ }^{2-4}$ The reported dip at the Rayleigh angle follows from the heuristic argument given above as well. In Fig. 3(b) the wave propagates a distance $\Delta_{s}$ in a lossy medium, and therefore this wave will have smaller amplitude at the receiver compared to the case shown in (a).

The solid curve in Fig. 4 is a plot of the reflection coefficient peak value as a function of frequency for the water-YAG interface. For this case $\theta_{R}=19.09^{\circ}$ and $\Delta_{s}=81.04 \lambda_{0}$. Hence the water path difference is $\Delta_{s} \sin \theta_{R}$ $=26.5 \lambda_{0}$. Our argument predicts a peak amplitude

$$
\exp \left(\frac{\alpha}{f^{2}} f^{2} \Delta_{s} \sin \theta_{R}\right)=\exp \left(8.79 \times 10^{-10} f\right)
$$

This is also plotted in Fig. 4 as a dashed line. The agreement between the curves at low frequencies supports the simple explanation mentioned above. At higher frequencies, expression (5) loses its validity since it is true for low loss cases only.

The peak amplitude goes to infinity at some critical frequency. This frequency is analogous to the "frequency of zero reflection" observed on lossy solids. Our argument tells us that at this frequency the Schoch dis-

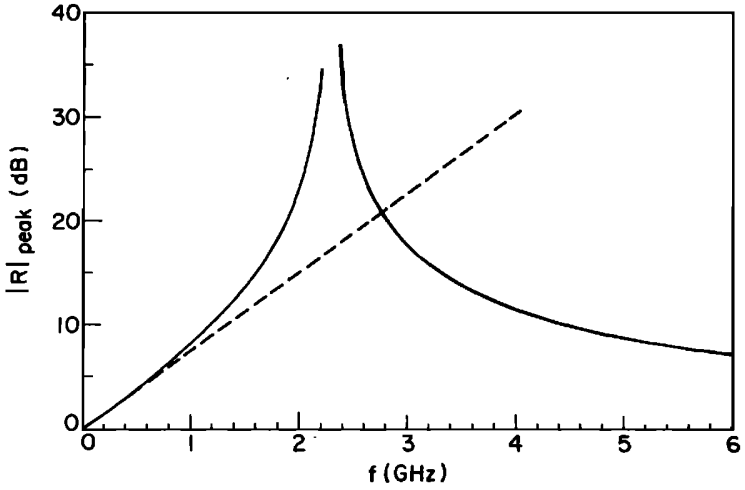

FIG. 4. The value of the reflection coefficient amplitude at the Rayleigh angle (solid line) for $\mathrm{H}_{2} \mathrm{O}-\mathrm{YAG}$ interface as a function of frequency. Dashed line is an approximation to this curve valid at low frequencies.

placement is infinite. That is to say, for some critical attenuation the Rayleigh wave will not leak back into the liquid. This may seem to violate the reciprocity principle. However the reflection coefficient peak at this frequency is infinitely narrow. In other words one has to use infinitely wide beams (or plane waves) to excite the Rayleigh wave. Thus, infinite power is involved and the reciprocity principle does not apply. Nevertheless, one can conclude that if a Rayleigh wave is excited by some other means it will not leak into the liquid provided the attenuation in the liquid is at that critical value.

For higher frequencies the peak amplitude decreases to approach to unity (see Fig. 4), suggesting that for a very lossy liquid the Schoch displacement would not be present.

\section{CONCLUSIONS}

Theoretical calculations show that the reflection coefficient for a lossy liquid-lossless solid interface has a peak at the Rayleigh angle. This peak value is greater than unity and its presence is explained by the Schoch displacement. The magnitude of the peak depends on the liquid attenuation and it goes to infinity at some critical attenuation value. The proposed physical explanation predicts that at that critical point, a Rayleigh wave propagating on the solid surface will not leak into the liquid.

\section{ACKNOWLEDGMENTS}

The author wishes to thank Professor C. F. Quate for a critical reading of this manuscript, and J. Fraser and V. Jipson for helpful discussions. This work was supported by the Air Force Office of Scientific Research. 
${ }^{1}$ L. M. Brekhovskikh, Waves in Layered Media (Academic, New York, 1960).

${ }^{2}$ V. M. Merkulova, Sov. Phys.-Acoust. 15 (3), (1970).

${ }^{3}$ G. Mott, J. Acoust. Soc. Am. 50, 820 (1971).
${ }^{4}$ F. L. Becker and R. L. Richardson, J. Acoust. Soc. Am. 51, 1610 (1972).

5 J. Attal and C. F. Quate, J. Acoust. Soc. Am. 59, 69 (1976).

${ }^{6}$ A. Schoch, Ergeb. Exact. Naturwiss. 23, 127 (1950). 